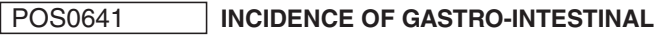 COMPLICATIONS IN RHEUMATOID ARTHRITIS PATIENTS RECEIVING BIOLOGICAL DMARDS IN OBSERVATIONAL COHORT STUDIES: A SYSTEMATIC LITERATURE REVIEW AND META-ANALYSIS}

A. Charlotte ${ }^{1}$, D. Jerome ${ }^{2}$, C. Lukas ${ }^{3}$, C. Rempenault ${ }^{3}$, A. Constantin ${ }^{4}$, J. Morel ${ }^{3}$. 'Lapeyronie Hospital, Montpellier, Rheumatology Department, Montpellier, France; ${ }^{2}$ Purpan University Hospital and University Toulouse III - Paul Sabatier, Rheumatology Department, Montpellier, France; ${ }^{3}$ Lapeyronie Hospital, Montpellier, Rheumatology Department, Montpellier, France; ${ }^{4}$ Purpan University Hospital and University Toulouse III - Paul Sabatier, Rheumatology Department, Toulouse, France

Background: Rheumatoid arthritis (RA) patients are at increased risk of gastro-intestinal (GI) perforations compared with non-RA patients, resulting in increased mortality. Clinical trials, post-marketing studies and registries have reported an increased risk of Gl perforations in RA patients treated with tocilizumab.

Objectives: The aim of our study was to assess the incidence of Gl complications among RA patients receiving bDMARDs in observational cohort studies Methods: A systematic literature review was carried out through September 2020 on the Pubmed, Embase and international congress databases, selecting observational cohort studies assessing the incidence of GI complications, including perforations and diverticulitis, in RA patients receiving bDMARDS. Keywords were "gastrointestinal perforation," "gastrointestinal disease", "diverticulitis", "biological DMARDs" and "rheumatoid arthritis" with no publication date limit. Studies were selected independently by two readers. Data were extracted by one investigator and independently checked by another. A meta-analysis was performed with Review Manager Software, with random-effects models, whenever methodologically possible and relevant.

Results: The literature search revealed 232 articles and abstracts of potential interest, and further examination resulted in 7 studies fulfilling required criteria. Among bDMARDs, Tocilizumab was associated with an increased incidence of GI perforations, with an overall incidence of 2.40 per 1000 person-years $(95 \%$ confidence interval $[95 \% \mathrm{Cl}] 1.45-3.35)$. The overall incidences of Gl perforations were 1.01 per 1000 PY [0.75-1.27] for TNF inhibitors, 1.07 per $1000 \mathrm{PY}$ [0.53-1.62] for abatacept and 1.12 per 1000 PY [0.16-2.08] for rituximab (Figure 1). In RA patients treated with tocilizumab, most of the perforations were located in the lower Gl tract, with an incidence of 2.24 per 1000 PY [1.243.52]. The incidences of upper GI perforations were similar across the different bDMARDs. The incidences of diverticulitis were 4.99 per 1000 PY [4.08-5.99] in RA patients receiving tocilizumab and 1.81 per 1000 PY [1.47-2.19] in those receiving TNF inhibitors.

Conclusion: In our meta-analysis, focused in RA patients receiving bDMARDs in observational cohort studies, tocilizumab was associated with an increased incidence of Gl perforations, mainly located in the lower Gl tract. An history of diverticulitis and long-term corticosteroid therapy were associated with an increased risk of $\mathrm{Gl}$ perforations.

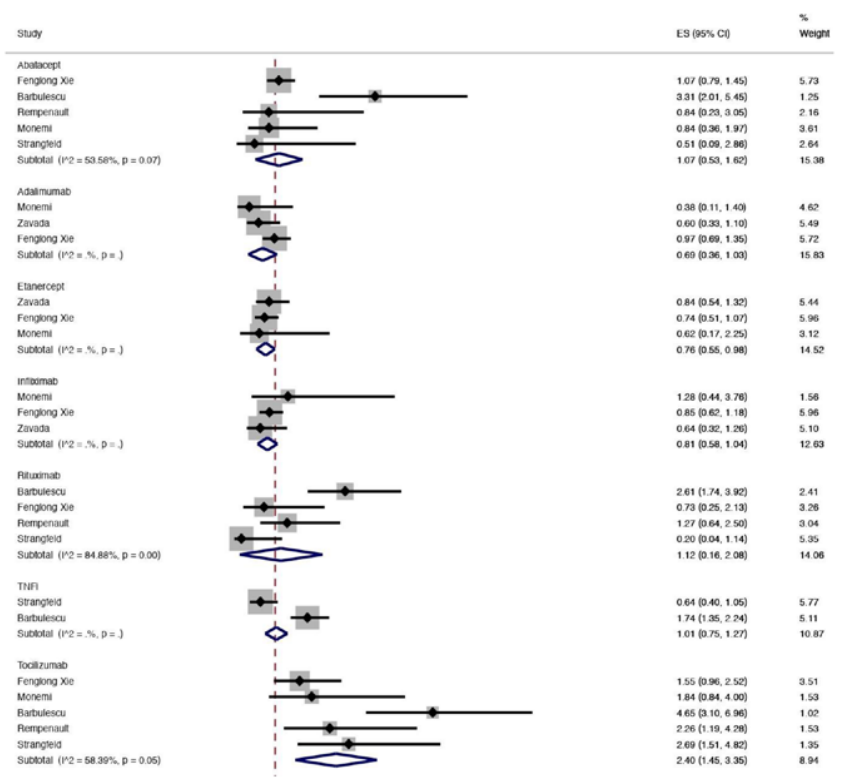

Figure 1. Meta-analysis of the incidences of gastro-intestinal perforations in RA patients receiving bDMARDs in observational cohort studies
Acknowledgements: We cannot express enough thanks to PhD Constantin and $\mathrm{PhD}$ Morel for their support and encouragement.

We would like to address a special word of thanks to PhD Lukas for his accuracy. Special Thanks to Claire Rempenault for her precious advices. You have been a role model for us.

Disclosure of Interests: None declared

DOI: 10.1136/annrheumdis-2021-eular.3755

\section{POS0642 THE IMPACT OF AGE ON DISCONTINUATION OF BIOLOGIC DMARDS IN PATIENTS WITH RHEUMATOID ARTHRITIS}

V. Rivera Teran ${ }^{1}$, S. Sicsik ${ }^{1}$, D. Vega-Morales ${ }^{2}$, F. Irazoque-Palazuelos ${ }^{1}$, D. Miranda ${ }^{2}$, J. C. Casasola ${ }^{1}$, S. Carriloº ${ }^{2}$ A. Peña ${ }^{1}$, A. Castillo Ortiz ${ }^{2}$, O. E. Muñoz-Monroy ${ }^{1}$, S. Duran Barragan² ${ }^{2}$ A. Paz ${ }^{1}$, E. Torres Valdéz ${ }^{2}$, L. F. Valdés Corona ${ }^{1}$, D. X. Xibille Friedmann ${ }^{2}$, E. Zamora ${ }^{1}$, A. Ramos ${ }^{2}$, N. Santana ${ }^{1}$, M. Vazquez ${ }^{2}$, F. Guerrero ${ }^{1}$, C. Zepeda ${ }^{2}$, K. Alvarado ${ }^{1}$, M. Rivera ${ }^{2}$, D. AlpizarRodriguez ${ }^{1}$ on behalf of Mexican Registry of Adverse Events involving Biological Therapies in Rheumatic Diseases (BIOBADAMEX). ${ }^{1}$ Colegio Mexicano de Reumatología, Unidad de Investigación, Mexico City, Mexico; ${ }^{2}$ Colegio Mexicano de Reumatología, Unidad de Investigación, Ciudad de México, Mexico

Background: Rheumatoid arthritis (RA) is the most common autoimmune disease. Older patients treated with biologic DMARDs (bDMARDs) are at a significantly greater risk of adverse effects (AEs) [1]. However, the rate of drug discontinuation because of adverse effects caused by bDMARDs has not differed in elderly compared to younger patients in different registries.

Objectives: Determine if drug discontinuation of bDMARDs differs by age in patients with rheumatoid arthritis in the Mexican Adverse Events Registry (BIOBADAMEX).

Methods: BIOBADAMEX is a Mexican ongoing cohort of patients using bDMARDs since 2016. In this analysis we included all patients with diagnosis of RA with at least two assessments. Survival on bDMARDs was estimated using Kaplan-Meier analysis. Predictors of discontinuation, including age older than median age in the sample were investigated by Cox regression analyses.

Results: Among 743 patients in the registry, 497 had RA diagnosis, from which, 214 had at least two assessments. At baseline, patients had a median (IQR) age of 53.4 (45-61) years old, median disease duration of 10.7 (6-17) months and median DAS28 of 4.7 (3-6). Conventional DMARDS were used by $185(87 \%)$ patients and 94 (44\%) patients used corticosteroids. Comorbidities were present in $194(91 \%)$. The most common bDMARDs received at baseline were abatacept $59(27 \%)$, tocilizumab $45(21 \%)$, adalimumab $31(15 \%)$ and certolizumab $30(14 \%)$. At the time of analysis, the median bDMARDs treatment duration was 21.0(13-34) months, 128 (59\%) had discontinued treatment, 66 for inefficacy, 32 for adverse events and 30 for others. Fig 1 shows discontinuation rate curves in patients younger and older than median age. Cox proportional-hazards demonstrated no significant differences regarding age older than median age (HR 1.1, $95 \% \mathrm{Cl} 0.8-1.4, \mathrm{p}=0.7$ ), female sex (HR 1.2, 95\% Cl 0.7-1.9, $\mathrm{p}=0.44$ ), use of corticosteroids (HR 1.2, 95\% Cl 0.9-1.6, $\mathrm{p}=0.20$ ), comorbidities (HR 0.9, 95\% 0.6-1.5, $\mathrm{p}=0.78$ ), DAS28 (HR 0.9, 95\% 0.9-1.1, $\mathrm{p}=0.93$ ) or other factors.

Conclusion: This analysis did not show a role of age on discontinuation of bDMARDs in Mexican RA patients. Further longitudinal analyses will be performed including more patients to assess retention rate of bDMARDs and identify predictive variables of discontinuation in Mexican population.

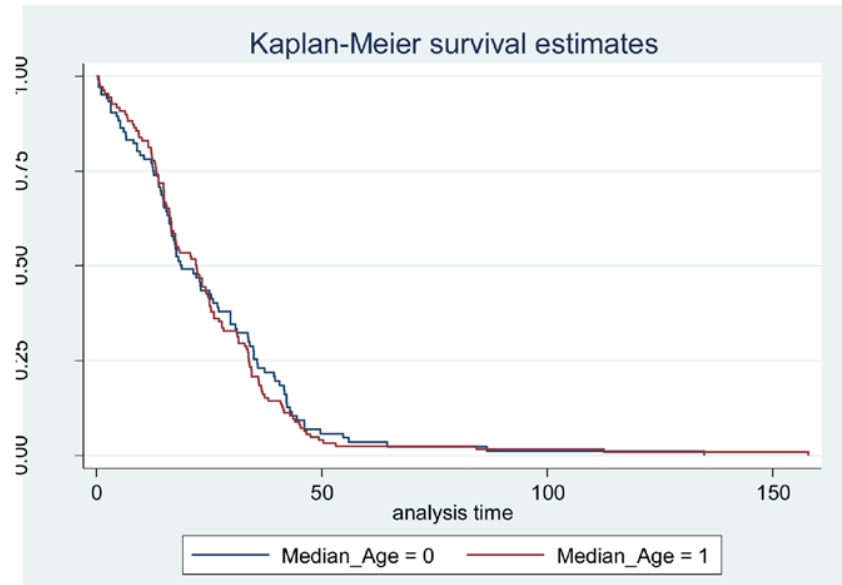

Figure 1. Discontinuation rate curves in patients younger and older than median age $(<53.4$ and $>=53.4$ years old) 